\title{
Diagnostic Value of Noninvasive Cardiac Function Measurement for Hypertensive Disorders in Pregnancy
}

\author{
Shifeng $\mathrm{Li}^{*}$, Xiaoshu Li², Liantao Nie1, Jihong Shen1, Yongsheng Wang1, Zhongjian $\mathrm{Li}^{1}$ \\ ${ }^{1}$ Electrocardiogram Lab, The Second Affiliated Hospital of Zhengzhou University, Zhengzhou, China \\ ${ }^{2}$ Department of Obstetrics and Gynecology, The Second Affiliated Hospital of Zhengzhou University, \\ Zhengzhou, China \\ Email: pladrli@163.com
}

Received 13 January 2015; accepted 23 March 2015; published 26 March 2015

Copyright (C) 2015 by authors and Scientific Research Publishing Inc.

This work is licensed under the Creative Commons Attribution International License (CC BY). http://creativecommons.org/licenses/by/4.0/

(c) (i) Open Access

\begin{abstract}
This paper aims to explore the diagnostic value of noninvasive cardiac function measurement for hypertension in pregnancy. One hundred and fifty-eight cases of gestational hypertension in our hospital were analyzed retrospectively while 30 healthy pregnant women were served as normal controls. The heart function parameters in the two groups were measured with noninvasive cardiac function analyzer, such as myocardial contractive force indexes (JP, VP), impedance indexes (RTM, RS), volume load indexes (GCV, LVDP) and left heart output index (LCI). In all 158 cases of gestational hypertension, the myocardial contractive force indexes, impedance indexes and left heart output index increased unusually. The difference between the two groups was statistically significant $(P<0.05)$. The myocardial contractive force indexes and the impedance indexes increased significantly higher than left heart output index $(P<0.05)$. The noninvasive cardiac function measurement was much valuable in the early diagnosis of pregnancy-induced hypertension, especially the myocardial contractive force indexes, impedance indexes and left heart output index, it appeared to be simple and efficient, and it deserves to be clinically popularized and applied.
\end{abstract}

\section{Keywords}

Hypertension in Pregnancy, Noninvasive Cardiac Function, Heart Function Parameters

\section{Introduction}

Hypertensive disorders in pregnancy are leading causes of maternal, fetal and neonatal morbidity and mortality

\footnotetext{
*Corresponding author.
}

How to cite this paper: Li, S.F., Li, X.S., Nie, L.T., Shen, J.H., Wang, Y.S. and Li, Z.J. (2015) Diagnostic Value of Noninvasive Cardiac Function Measurement for Hypertensive Disorders in Pregnancy. Open Journal of Obstetrics and Gynecology, 5, 181-184. http://dx.doi.org/10.4236/ojog.2015.54025 
worldwide. A recent epidemiological investigation in China involving 25 provinces showed that the average morbidity of hypertensive disorders in pregnancy was 9.4\%. Early diagnosis and correct therapy are important to decrease the mortality and morbidity of the pregnancy and newborns. However, the present study in this field mainly focuses on the etiology, and little research has been done to study the changes of cardiac function in these patients. This population-based study explored the cardiac function of 158 cases with hypertensive disorders in pregnancy using noninvasive cardiac function detector, aiming to explore a simple and quick way to evaluate cardiac function and detect hypertensive disorder in pregnancy at an early stage.

\section{Methods}

\subsection{Patients}

The study population consisted of 128 cases of pregnant women who had been diagnosed to be hypertensive disorders in pregnancy form out-patient and in-patient in our hospital, including 40 severe preeclampsias and eclampsias cases as the severe group and 88 mild preeclampsias cases as the slight group. 30 cases of normal pregnant women were set as the control group. All cases had no chronic hypertension, diabetes and kidney disease. The age ranged from 20 to 36 years old, and the average age was 30.9. The gestational weeks ranged from 30 to 42 weeks, and the average gestational weeks were 37.4 .

\subsection{Diagnostic Criteria}

The classification of HDCP is difficult because of limited knowledge about its etiology and the lack of conformity of definitions [1]. The diagnostic criteria in our study were based on the definitions made by WHO in 1987 and further amendments by the American College of Obstetricians and Gynaecologists [2]. The hypertensive pregnancy disorders were stratified into gestational hypertension, mild preeclampsia, and severe preeclampsia (including eclampsia and hemolysis, elevated liver enzymes, and low platelets syndrome).

\subsection{Instrument and Observed Parameters}

The heart function parameters in two groups were measured by noninvasive cardiac function analyzing machine, that is the QXG-VIA Type synchronous left and right cardiac function analyzer made by Hangzhou Gaolian Medical Instrument Factory in China. The cardiac function of 128 HDCP cases was detected and the following parameters were observed:

1) Force parameters of the myocardium; $J P$-the ejection blood pressure of left ventricular, Normal range: (2.03 - 2.53) (N/CM $\left.{ }^{2}\right)$; VP-the effective pump power of left ventricular, Normal range: $\left(1.58\right.$ - 1.98) $\left(\mathrm{N} / \mathrm{CM}^{2}\right)$.

2) Impedance parameters of the heart; $R T M$-the total impedance, Normal range: $\left(1121\right.$ - 1593) $\left(\mathrm{G} / \mathrm{CM}^{4} / \mathrm{S}\right)$; $R S$-the artery characteristic impedance, Normal range: (169.3 - 201.9) $\left(\mathrm{G} / \mathrm{CM}^{4} / \mathrm{S}\right)$.

3) Volume load parameters of the heart; GCV-the effective circulating blood volume, Normal range: (2238 3339) (ML); LVDP-the left ventricular end-diastolic pressure, Normal range: (3.3 - 10.3) (MMHG).

4) Output parameters of the left heart; LCI-left cardiac index, Normal range: $(2.43-4.15)\left(\mathrm{L} / \mathrm{MIN} / \mathrm{M}^{2}\right)$.

\subsection{Statistical Analysis}

All analyses were conducted using SPSS Version 10.0. The data are expressed as mean \pm standard deviation (SD). Statistical analysis between groups was assessed by independent-samples t test. A P $<0.05$ was considered statistically significant.

\section{Results}

Cardiac function parameters of the 128 HDCP cases of increased in varying degrees. The force parameters, impedance parameters and output parameters of the severe group and the mild group is abnormally high, and the comparison with the control group had statistically significant difference $(\mathrm{P}<0.05)$. The volume load parameters in the severe group was obviously higher than the control group, and the difference was statistically significant $(\mathrm{P}<0.05)$ (Table 1$)$.

\section{Discussion}

Criteria established by the National High Blood Pressure Working Group, in pregnant women, gestational hyper- 
Table 1. Heart function parameters of 128 cases of hypertensive disorders in pregnancy.

\begin{tabular}{ccccc}
\hline \multirow{2}{*}{ Heart function parameters } & & \multicolumn{2}{c}{ HDCP group $^{*}$} & Control group $^{*}$ \\
\cline { 3 - 4 } & & The severe group & The mild group & \\
\hline \multirow{2}{*}{ The force parameters } & JP & $2.79 \pm 0.23$ & $2.98 \pm 0.31$ & $2.03-2.53$ \\
& VP & $2.23 \pm 0.21$ & $2.42 \pm 0.29$ & $1.58-1.98$ \\
The impedance parameters & RTM & $1714 \pm 230$ & $2014 \pm 310$ & $1121-1593$ \\
The output parameters & RS & $246.2 \pm 23.1$ & $265.8 \pm 27.3$ & $161-202$ \\
The volume load parameters & LCI & $4.23 \pm 0.64$ & $4.56 \pm 0.56$ & $2.43-4.15$ \\
& GCV & $3448 \pm 210$ & $4642 \pm 240^{\Delta}$ & $2238-3339^{\Delta}$ \\
\hline
\end{tabular}

Notes: ${ }^{*}$ comparision between two groups $\mathrm{P}<0.05,{ }^{\triangle}$ comparision between two groups $\mathrm{P}<0.05$.

tension is defined as: $\mathrm{SBP} \geq 140 \mathrm{~mm} \mathrm{Hg}$ or DBP $\geq 90 \mathrm{mmHg}$ that occurs after 20 weeks of gestation in a woman with previously normal blood pressure [3]. The etiology and pathophysiology of HDP remains unexplained. This may be due to the heterogeneous nature of HDP and its varied clinical progression. Pathogenesis may also differ according to the presence of risk factors and the timing of disease onset [4]. Pregnancy is considered a state of "gestational hypervolemia" as it is hallmarked by a vasodilatory state that leads to increased urinary $\mathrm{Na}^{+}$ and water retention. The systemic vascular resistance (SVR) decreases significantly in pregnancy as well [5].

The basic pathogenesis is the consequence of a general vascular disturbance which leads to edema and spasm of arterioles and viscera ischemia. HDCP patients are in the state of lower output-high resistance, the spasm of arterioles may result in myocardial damage, thus it is very important to identify HDCP in the early stage. But it is not easy to identify HDCP because of its varied clinical features. We used noninvasive cardiac function analyzing machine for early diagnosis of HDCP. The QXG-VIA Type synchronous left and right cardiac function analyzer based on the theory and technology of noninvasive surface signal detection. It was made according to biology cardiovascular elasticity model theory and analyzes the circulatory system by state space method. It can fully evaluate the cardiac function from four aspects (time phase, pressure, capacity and impedance) and reflect the changes of heart function in HDCP patients dynamically and quantitatively [6]. It is meaningful to investigate the effect of change in artery blood flow on heart structure and heart function in patients with pregnancy induced hypertension disorders [7]. In the mild preeclampsia as group, the patients were in the state of lower output-high resistance, so the force parameters, impedance parameters and output parameters parameters that reflected left heart function increased abnormally, while the volume load parameters increased little. In the severe preeclampsia and eclampsia group, the volume load parameters were apparently unusual because of the water and sodium retention on the basis of the abnormal of the cardiac function parameters in the mild group.

The etiology of preeclampsia is unclear, which $t$ brings huge difficulties to the treatment. Many trials has been conducted but they were small and of uncertain quality, and did not report potential adverse effects of rest, nor women's views or perceptions of impact on quality of life [8]. The current treatments focus on the symptomatic treatment and prevention of severe preeclampsia and eclampsia to decrease the mortality and morbidity of the pregnancy and perinatal. The basic principles for treatment were individual therapy according to the serious degree and classification of the clinical condition. Disease variety in antepartum, intrapartum and postpartum surgery is impartment to monitor and evaluate the treatment effect closely because of the complication of condition. The noninvasive cardiac function analyzing machine can help to observe dynamically the cardiac function changes by detecting the cardiac function parameters. Then some medicines which have no effect on the pregnancy and perinatal such as calcium antagonists and $\beta$ receptor antagonists can be selected for treatment. The capacity expansion medicine can be selected according to load parameters.

\section{Study Strengths and Limitations}

We found that the force parameters, the impedance parameters and the left cardiac output parameters of HDCP were abnormally high through the noninvasive heart function analyzer. We should pay more attention to the early diagnosis of HDCP in clinic, and concern the population with high risk factors of HDCP in cardiovascular 
risk stratification, such as the age, blood glucose, lipid metabolism disorders, fat, high salt, insomnia and history of HDCP according to the classification approaches developed by the Joint National Committee on Prevention, Detection, Evaluation, and Treatment of High Blood Pressure (JNC VI) [9] and the World Health Organization and the International Society of Hypertension (WHO-ISH) [10]. We should do ambulatory blood pressure monitoring or home blood pressure monitoring for high-risk mothers. Besides, periodical noninvasive cardiac function tests were also necessary to help find out HDCP and take effective intervention measures. It is helpful to reduce the damage of target organ, reduce prevalence of preeclampsia and eclampsia, and improve the prognosis of pregnancy and perinatal.

\section{Acknowledgements}

We are deeply indebted to Professor Zhi-guang Ping from Zhengzhou University, whose concepts, analysis, and reflections were the foundations for this work. We thank the cooperation with the department of Obstetrics and Gynecology.

\section{References}

[1] AbouZahr, C. and Guidotti, R. (1998) Hypertensive Disorders of Pregnancy. In: Murray, C.J.L. and Lopez, A.D., Eds., Health Dimensions of Sex and Reproduction: The Global Burden of Sexually Transmitted Diseases, Maternal Conditions, Perinatal Disorders, and Congenital Anomalies, WHO, Geneva.

[2] National High Blood Pressure Education Program Working Group on High Blood Pressure in Pregnancy (2000) Report on the National High Blood Pressure Education Program Working Group on High Blood Pressure in Pregnancy. American Journal of Obstetrics and Gynaecology, 183, s1-s22. http://dx.doi.org/10.1067/mob.2000.107928

[3] ACOG Committee on Practice Bulletins-Obstetrics (2002) American College of Obstetricians and Gynecologists (ACOG) Practice Bulletin. Diagnosis and Management of Preeclampsia and Eclampsia. Number 33, January 2002. Obstetrics and Gynecology, 99, 159-167.

[4] Sibai, B., Dekker, G. and Kupferminc, M. (2005) Pre-Eclampsia. The Lancet, 365, 785-799. http://dx.doi.org/10.1016/S0140-6736(05)71003-5

[5] Dildy, G. (2004) Critical Care Obstetrics. 4th Edition, Blackwell Science, Oxford. http://dx.doi.org/10.1002/9780470760086

[6] Roberts, J.M. and Hubel, C.A. (2009) The Two Stage Model of Preeclampsia: Variations on the Theme. Placenta, 30, S32-S37.

[7] Li, Y., Du, L. and Ma, C.-E. (2001) Change of Left Heart Function and Atrioventricular Structure in Patients with Pregnancy Induced Hypertension Syndrome. Current Advances in Obstetrics and Gynecology, 10, 42-43.

[8] Meher, S. and Duley, L. (2006) Rest during Pregnancy for Preventing Pre-Eclampsia and Its Complications in Women with Normal Blood Pressure. Cochrane Database of Systematic Reviews, 6, Article ID: CD005939.

[9] The Sixth Report of the Joint National Committee on Prevention, Detection, Evaluation, and Treatment of High Blood Pressure. (1997) Archives of Internal Medicine, 157, 2413-2446. [Erratum, Archives of Internal Medicine, 1998, 158, 573.] http://dx.doi.org/10.1001/archinte.1997.00440420033005

[10] Guidelines Subcommittee (1999) World Health Organization-International Society of Hypertension Guidelines for the Management of Hypertension. Journal of Hypertension, 17, 151-183. 